\title{
A robust multi-scale gapped smoothing algorithm for baseline-free damage mapping from raw thermal images in flash thermography
}

\author{
by Gaétan Poelman*, Saeid Hedayatrasa*,**, Joost Segers*, Wim Van Paepegem* and \\ Mathias Kersemans*
}

\author{
* Mechanics of Materials and Structures (UGent-MMS), Department of Materials, Textiles and Chemical \\ Engineering (MaTCh), Ghent University, Technologiepark-Zwijnaarde 46, 9052 Zwijnaarde, Belgium, \\ Gaetan.Poelman@UGent.be \\ ** SIM Program M3 DETECT-IV, Technologiepark-Zwijnaarde 48, B-9052 Zwijnaarde, Belgium
}

Corresponding Author: Gaétan Poelman, Email: Gaetan.Poelman@UGent.be

\begin{abstract}
Flash thermography is a promising non-destructive testing technique for the inspection of composite components. However, non-uniform heating, measurement noise and lateral heat diffusion complicate the interpretation of thermographic measurements. In order to overcome these difficulties, a novel baseline-free processing technique called 'Multi-Scale Gapped Smoothing Algorithm' is presented. This algorithm constructs a damage map directly from the measured data, in which an (almost) zero-reference background is obtained, and where measurement noise and excitation non-uniformity are effectively suppressed. The efficiency of the proposed technique is evaluated and confirmed through synthetic data and experimental results of a carbon fiber reinforced polymer with various artificial defects.
\end{abstract}

\section{Introduction}

Composites are materials that exhibit advantageous properties in comparison to traditional metals, such as their high stiffness-to-weight ratio and good corrosion resistance. On the other hand, their layered structure makes that these materials are susceptible to internal damages, which may arise either during manufacturing or during in-service loading. These damage features may compromise the component's integrity, and must therefore be detected as early as possible and in a non-destructive manner.

Optical infrared thermography (IRT) is a promising non-destructive testing (NDT) technique which provides a fast, full-field, safe, and non-contact inspection [1,2]. With this technique, the mismatch in thermal properties between the sound material and internal defects is exploited for defect detection. An optical source is used to provide an external stimulus which causes thermal waves to propagate into the material's thickness, while in the meantime a high-sensitivity infrared (IR) camera monitors the excited surface's thermal evolution. An internal defect, characterized by different thermal properties than the surrounding sound material, causes localized changes in the heat propagation, which can be detected in the thermographic recordings. The most-commonly used approaches are lock-in thermography [3], in which a harmonically modulated signal is used for the optical excitation, and flash thermography (FT) [4], where the sample's cooling down regime is evaluated after being excited with a high-intensity flash. Optical IRT is inherently limited due to the diffusive and highly damped nature of the induced thermal waves, making the detection of deep and small defects a challenging task $[1,2]$.

In order to improve a defect's detectability, a wide variety of processing algorithms have been developed over the past decades. Some of the most commonly implemented data processing approaches are pulsed phase thermography (PPT) [5], principal component thermography (PCT) [6], thermographic signal reconstruction (TSR) [7] and dynamic thermal tomography (DTT) [8]. Besides data processing techniques, which analyse the entire thermographic sequence, image processing techniques also work towards enhanced defect detectability, however, they are only fed the information that is available in one thermogram. Well-known examples of such image enhancement techniques are the median filter (used to remove spiky features) and the Sobel filter (for edge extraction). Recently, an alternative image processing technique called gapped smoothing algorithm (GSA), which originated in the field of vibrational testing [9, 10], was introduced as a baseline-free technique for optical IRT, and showed promising results by obtaining an enhanced defect detectability and an almost-zero reference background [11]. Additionally, it was observed in that study that the size of the evaluated spatial gap has a direct influence on the defect detectability. Since the standard GSA implements a fixed spatial gap size, it experiences difficulties when it is applied on IRT datasets, which typically contain non-uniform heating, lateral heat diffusion and measurement noise.

This contribution discusses the Multi-Scale Gapped Smoothing Algorithm (MSGSA) [12], which is a novel image processing technique that evaluates a wide range of spatial gap sizes and combines them into a unique damage index map. This multi-scale approach robustly detects defects of different sizes in the presence of non-uniform background and measurement noise. The remainder of the paper is organized as follows: section 2 presents a brief case study on 1D synthetic data, indicating the challenges and shortcomings of the standard GSA. Next, the novel MSGSA is introduced in section 3 , and its performance is demonstrated on the 1D synthetic data. In section 4, MSGSA is applied as a 2D image 
processing technique on a raw thermogram from a flash thermographic experiment. Lastly, section 5 gathers the main conclusions.

\section{Gapped Smoothing Algorithm (GSA)}

The gapped smoothing algorithm (GSA) estimates the behavior of a sample in the undamaged state from a measurement in its damaged state. In the original GSA implementation [9], a single pixel is considered for the spatial gap, and its sound behavior is approximated by fitting a cubic polynomial to the gapped pixel's surrounding pixels. However, considering the typical shape of IRT measurements, it was found that a linear fitting polynomial is more appropriate for thermographic datasets [12]. Besides the obvious reduction in the polynomial's order, this also accounts for a large reduction in calculation time. In the following, the size of the spatial gap is extended, and the effect on the defect detectability is studied.

The working principle of GSA is explained by first introducing a 1D synthetic dataset $T$ that contains $n$ datapoints (i.e. $n$ pixels), where $T\left(x_{i}\right)$ is the temperature at the $i^{\text {th }}$ pixel, located at $x_{i}$. For the original implementation, where the spatial gap contains one pixel, any individual pixel $x_{i}$ is replaced with the mean temperature value of its left and right neighboring pixels $x_{i-1}$ and $x_{i+1}$. For an increased spatial gap size of $k$ pixels, the spatial gap's central pixel $x_{i}$ is replaced by the mean temperature value of the left and right neighboring pixel groups (both containing $k$ pixels), adjacent to the spatial gap. Next, the contrast is calculated between the estimated sound behavior and the actual measured behavior, after which a dimensionless damage index can be calculated. For a detailed explanation of this procedure, the reader is referred to Reference [12].

The performance of the standard GSA, i.e. spatial gap size $k$ of 1 pixel, is evaluated on the 1D synthetic dataset in Figure 1(a), which represents a thermographic dataset with three defects of varying defect size and thermal contrast. The defects are modelled using Gaussian-like distributions to represent smooth defects (i.e. no abrupt changes), with amplitudes of $0.025,0.1$ and 0.05 for $D_{1}, D_{2}$ and $D_{3}$, respectively. A dominant background non-uniformity was added using a Gaussian distribution. Additionally, artificial measurement noise was added as white Gaussian noise with a standard deviation of 0.014 . The obtained damage index map by the standard GSA, i.e. spatial gap size $k$ of 1 pixel, is depicted in Figure 1(b), in which none of the defects can be detected. This is actually not a surprising result, considering that a spatial gap size of only one pixel is almost uniquely influenced by the random fluctuations of the measurement noise, rather than by the defect signatures.

Since a spatial gap size of 1 pixel is ineffective for thermographic measurements with background non-uniformity and measurement noise, it is of interest to investigate the effect of increasing the spatial gap size on a defect's detectability. Therefore, the damage index values for all pixels are calculated for a wide range of odd-valued spatial gap sizes $(k=$ $1,3, \ldots, 357)$, and are displayed in Figure 1(c). The areas between the white dashed lines and the edges contain the pixels for which the spatial gap sizes are too large to be evaluated properly, and are therefore omitted from the analysis. It is immediately clear from the figure that the evaluated spatial gap size has a significant effect on the damage index value, and that defects of different sizes behave differently. In accordance with Figure 1(b), it can be observed that the detectability for small gap sizes (see bottom rows of the figure) is limited due to the artificial measurement noise. The small defect $D_{1}$ has its highest damage index value at relatively small spatial gap sizes, while a larger defect such as $D_{2}$ is better detected at larger spatial gap sizes. Of course, intermediate defect sizes (e.g. $D_{3}$ ) fall in between defects $D_{1}$ and $D_{3}$. $A$ large defect $\left(D_{2}\right)$ has a wider range of appropriate spatial gap sizes (i.e. increased damage index values) than a smaller defect $\left(D_{1}\right)$. Notice that side lobes are obtained for the defects, which are a result of the calculation procedure: the damage index value is calculated by evaluating neighboring pixels, which results in the 'smearing' of a defect's signature [9]. The lateral extent of the side lobes increases with increasing spatial gap size, which means that a sensible upper limit should be chosen for the evaluated spatial gap sizes. For the largest defect $\left(D_{2}\right)$, only the edges are detected for relatively small spatial gap sizes (i.e. $k$ up to 75 pixels), while the entire defect is highlighted for larger spatial gap sizes. The strong background nonuniformity causes a slight increase in the damage index values in the region around defect $D_{1}$ for large spatial gap sizes. Thus, an upper limit on the spatial gap size is also required in order to limit the effects of the non-uniform background. Figure 1(d) plots the damage index values in function of the evaluated spatial gap size for the center pixels of the three defects and a sound pixel (indicated in Figure 1(c)). The damage index value of the sound pixel randomly fluctuates for small spatial gap sizes, after which it adopts a low (near-zero) damage index value for the remainder of the evaluated spatial gap sizes. Furthermore, this figure clearly illustrates that the optimal spatial gap size depends on the lateral size of the defect, with the optimal spatial gap being larger for bigger defects.

These results demonstrate that there exists an optimal spatial gap size for each defect, and that it is related to the defect's size. In an experimental environment, there is typically no a priori information about the internal defects and their sizes, which means that the selection of the optimal spatial gap size(s) to be evaluated is far from straightforward. In order to overcome this challenge, the authors have developed a Multi-Scale Gapped Smoothing Algorithm (MSGSA), which is introduced below. 
(a)

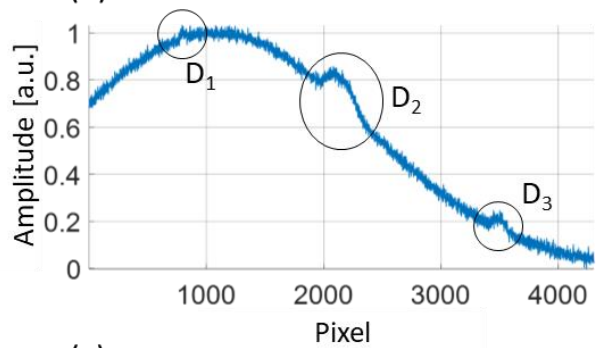

(c)

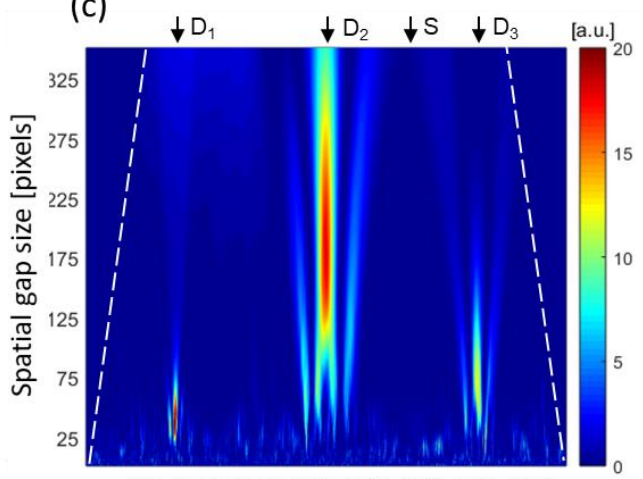

5001000150020002500300035004000 Pixel number (b)

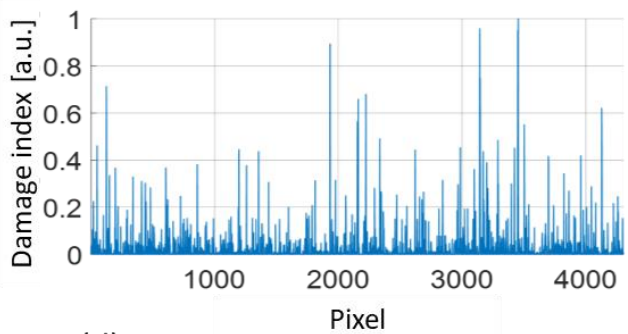

(d)

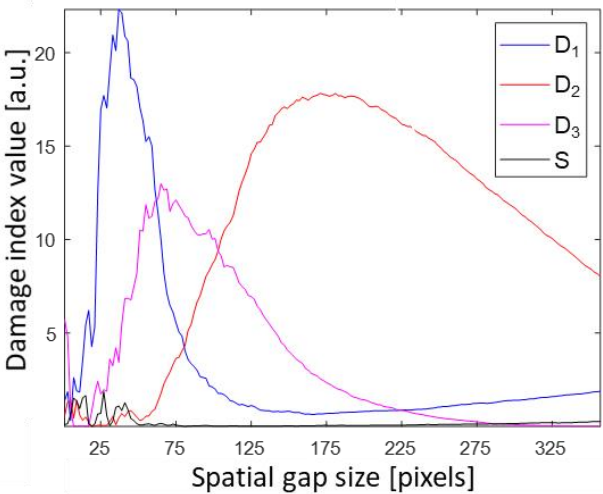

Figure 1: (a) 1D synthetic input dataset (with non-uniform background and noise); (b) Output of GSA; (c) Surface plot of the damage index value in function of the evaluated spatial gap size for the 1D synthetic input dataset in (a); (d) Damage index value in function of the evaluated spatial gap size for the defects' center pixel and a sound pixel.

\section{Multi-Scale Gapped Smoothing Algorithm (MSGSA)}

In order to avoid having to perform a careful pre-selection of the spatial gap sizes that need to be evaluated, MSGSA calculates damage index maps for a wide range of odd-valued spatial gap sizes $\left(k=1,3, \ldots, k_{\text {max }}\right)$. The upper limit for the evaluated spatial gap sizes $k_{\max }$ is defined by dividing the number of pixels in the input vector $n$ by a factor of 12 , which ensures that large defects are detected, while suppressing side lobes and background non-uniformity. Next, the different damage index maps are combined into a global damage index map through a weighted summation procedure that guarantees a major contribution of the most effective spatial gap sizes while limiting the contribution of less favorable spatial gap sizes [12].

The output of the MSGSA and standard GSA procedures on the synthetic 1D dataset with background nonuniformity and artificial measurement noise (see Figure 2(a)) are presented in Figure 2(b). The total calculation of the MSGSA procedure required $0.07 \mathrm{~s}$ on a laptop with Intel Core i7 CPU with 32 GB RAM. As was already observed, the standard GSA does not yield any defect detection. On the other hand, MSGSA (i) detects all three defects clearly, (ii) reduces the strong background non-uniformity to an (almost) zero-reference background and (iii) suppresses the measurement noise. Notice that the extent of the side lobes is proportional to the defects' lateral size. 
(a)

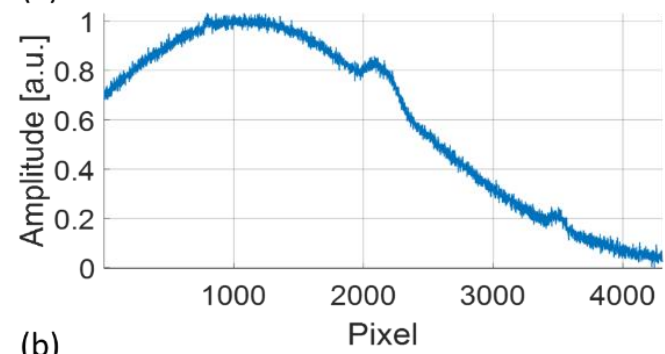

(b)

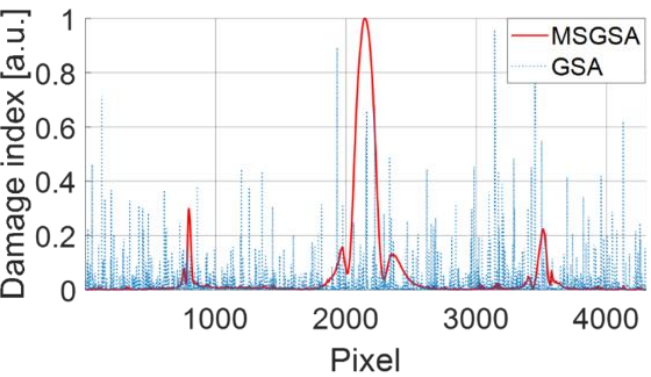

Figure 2: (a) Synthetic 1D dataset with non-uniform background and noise (see Figure 1(a)); (b) normalized output of GSA and MSGSA.

The MSGSA algorithm can be easily extended to operate on 2D images by applying MSGSA separately on each vector in the $\mathrm{X}$ - and $\mathrm{Y}$-direction, after which the damage index maps in $\mathrm{X}$ - and $\mathrm{Y}$-direction are combined into a global damage index map [12]. Maintaining the calculation procedure largely one-dimensional has the advantage that it calculates rapidly and that complex defect shapes (e.g. tilted defects) can be accurately detected, which would not be the case if the spatial gap would be extended towards a 2D shape.

\section{Experimental validation}

In this section, the performance of MSGSA is demonstrated on an experimentally generated dataset. Therefore, a CFRP sample with a total thickness of $2.9 \mathrm{~mm}$ and a uni-directional layup of $[0]_{10}$ is inspected through flash thermography. Artificial defects were introduced by inserting square ethylene-tetrafluorethylene (ETFE) inserts with lateral sizes of 5 , 10 and $15 \mathrm{~mm}$, and a thickness of $90 \mu \mathrm{m}$, at different depths (ranging from $0.25 \mathrm{~mm}$ to $1.5 \mathrm{~mm}$ ). A schematic illustration of the inspected CFRP sample is provided in Figure 3(a).

The inspected sample was excited using a Hensel linear flash lamp, which provides a flash of 5 ms that consumes $6 \mathrm{~kJ}$ of energy. The excited surface's temperature evolution was monitored using a FLIR A6750sc. This IR camera has a focal plane array of $640 \times 512$ cryo-cooled InSb detectors, which have a noise-equivalent differential temperature (NEDT) of $\leq 20 \mathrm{mK}$ and a bit depth of 14 bits. The camera is sensitive in the wavelength range of 3-5 $\mu \mathrm{m}$. The flash lamp was placed at a distance of $\sim 200 \mathrm{~mm}$ from the sample, while the IR camera was positioned $\sim 900 \mathrm{~mm}$ away. In order to introduce a significant amount of non-uniform heating, the flash lamp was purposely placed more on the right side of the sample. The temperature evolution was recorded at a framerate of $25 \mathrm{~Hz}$, after which the thermographic sequence was imported into MATLAB to perform the MSGSA processing.

The raw thermogram at $3 \mathrm{~s}$ after flash excitation is selected for the experimental validation of the MSGSA technique, and is presented in Figure 3(b). It is clear that the off-center placement of the flash lamp introduced a significant amount of background non-uniformity, which complicates the detection of the defects. The shallowest column of defects remains unidentified in this thermogram, and only a limited number of the deeper defects could be reliably identified in the absence of prior information. The global damage index map calculated by MSGSA is displayed in Figure 3(c), which required around $0.3 \mathrm{~s}$ to calculate. MSGSA enhances the overall defect detectability, making it possible to identify defects up to the fifth column, and successfully removes almost all of the background non-uniformity. Additionally, a localized horizontal disturbance can be observed just below the bottom row of inserts. The exact cause for this anomaly is not clear, however, careful visual inspection revealed that this region corresponds to some localized disturbances in the surface quality. 
(a)

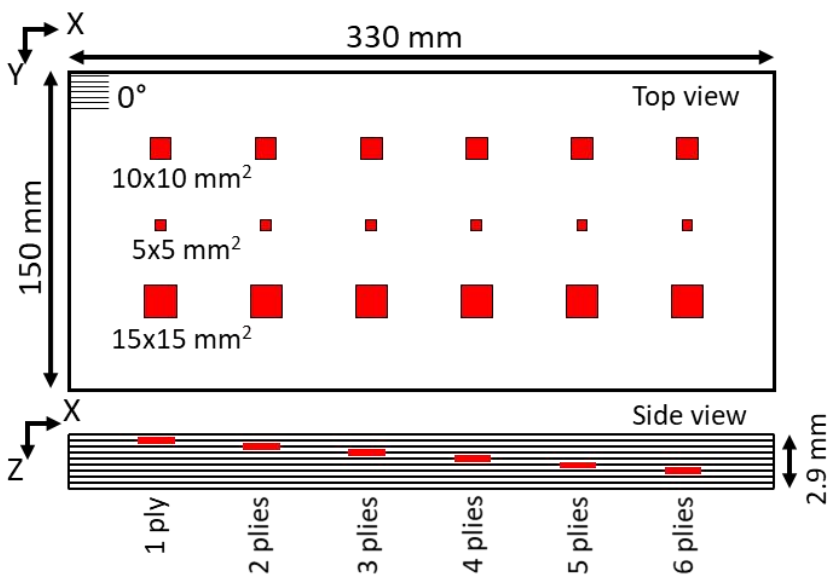

(b)

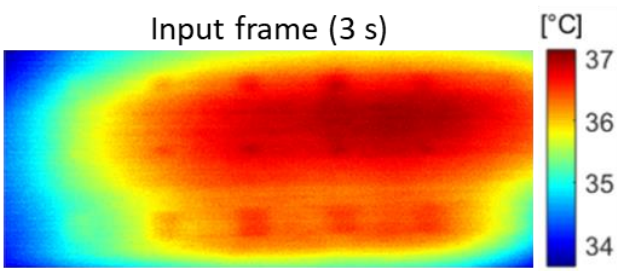

(c)

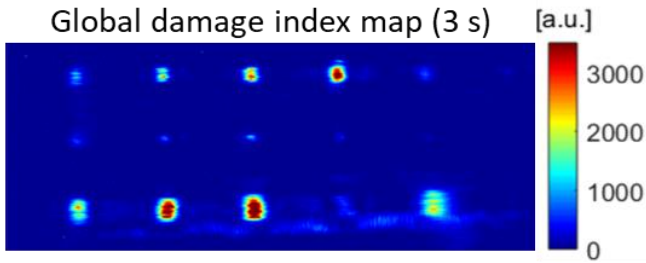

Figure 3: (a) Schematic illustration of the inspected CFRP sample with inserts; (b-c) Input frame and output of MSGSA for a thermogram at $3 \mathrm{~s}$ after flash excitation.

\section{Conclusion}

In this paper, a novel image processing technique called multi-scale gapped smoothing algorithm (MSGSA) is discussed for the baseline-free processing of noisy thermograms that suffer from a high level of background non-uniformity. The technique is based on the observation that a wide range of spatial gap sizes needs to be evaluated in order to robustly detect defects of different sizes. For an input thermogram, the MSGSA calculates a unique damage index map that provides an enhanced defect detectability, obtains an almost zero-reference level and effectively suppresses measurement noise.

The performance of MSGSA is first demonstrated on a synthetic 1D dataset that includes several artificial damages with highly different lateral sizes. Next, its robustness is experimentally validated for a CFRP sample with artificial defects by processing a challenging thermogram obtained through flash thermographic inspection. The results demonstrate that MSGSA is a robust image processing technique that manages to extract defects of highly different sizes, while also suppressing effects of non-uniform background and measurement noise. Lastly, while the performance of MSGSA has only been explicitly demonstrated for a flash thermographic dataset in this paper, the technique is also applicable for alternative heating procedures.

\section{Acknowledgements}

The authors acknowledge the SBO project DETECT-IV (Grant no. 160455), which fits in the SIM research program MacroModelMat (M3) coordinated by Siemens (Siemens Digital Industries Software, Belgium) and funded by SIM (Strategic Initiative Materials in Flanders) and VLAIO (Flemish government agency Flanders Innovation \& Entrepreneurship). The authors also acknowledge Fonds voor Wetenschappelijk Onderzoek Vlaanderen (FWOVlaanderen) through grants $1 \mathrm{~S} 11520 \mathrm{~N}, 1148018 \mathrm{~N}$ and $12 \mathrm{~T} 5418 \mathrm{~N}$.

\section{References}

1. Yang, R. and Y. He, Optically and non-optically excited thermography for composites: A review. Infrared Physics \& Technology, 2016. 75: p. 26-50.

2. Ciampa, F., et al., Recent Advances in Active Infrared Thermography for Non-Destructive Testing of Aerospace Components. Sensors (Basel), 2018. 18(2).

3. Busse, G., D. Wu, and W. Karpen, Thermal wave imaging with phase sensitive modulated thermography. Journal of Applied Physics, 1992. 71(8): p. 3962-3965.

4. Milne, J.M. and W.N. Reynolds, The non-destructive evaluation of composites and other materials by thermal pulse video thermography. Thermosense VII, 1985: p. 119-1222.

5. Maldague, X. and S. Marinetti, Pulse phase infrared thermography. Journal of Applied Physics, 1996. 79(5): p. 2694-2698.

6. Rajic, N. and DSTO-TR-1298, Principal Component Thermography. 2002, Defence Science and Technology Organisation Victoria (Australia) Aeronautical and Maritime Research Lab. p. 38.

7. Shepard, S.M., Temporal noise reduction, compression and analysis of thermographic data sequences. 2003, Thermal Wave Imaging, Inc.: US.

8. Vavilov, V.P. Dynamic Thermal Tomography: New NDE Technique to Reconstruct Inner Solids Structure Using Multiple IR Image Processing. in Review of Progress in Quantitative Nondestructive Evaluation. 1992. 
9. Ratcliffe, C.P., Damage detection using a modified Laplacian operator on mode shape data. Journal of Sound and Vibration, 1997. 204(3): p. 505-517.

10. Ratcliffe, C.P. and W.J. Bagaria, Vibration Technique for Locating Delamination in a Composite Beam. AIAA Journal, 1998. 36(6): p. 1074-1077.

11. Li, B., et al., Gapped smoothing algorithm applied to defect identification using pulsed thermography. Nondestructive Testing and Evaluation, 2015. 30(2): p. 171-195.

12. Poelman, G., et al., Multi-scale gapped smoothing algorithm for robust baseline-free damage detection in optical infrared thermography. NDT \& E International, 2020. 112. 